\title{
Long Survival in a Rare Case of Hepatocellular Carcinoma that Metastasized to the Ovary: A Case Report
}

Akimasa Fukui ${ }^{1 *}$, Kimio Ushijima1, Shin Nishio${ }^{1}$, Keizo Fujiyoshi ${ }^{1}$, Masayoshi Kage ${ }^{2}$ and Toshiharu Kamura ${ }^{1}$

${ }^{1}$ Department of Obstetrics and Gynecology, Kurume University School of Medicine, Japan

${ }^{2}$ Department of Pathology, Kurume University School of Medicine, Japan

\begin{abstract}
Hepatocellular carcinoma usually metastasizes to the lung, bone, adrenal glands, diaphragm and lymph nodes. In this study, we present a case of hepatocellular carcinoma that metastasized to the ovary. To the best of our knowledge, there are very few reports of such cases published in English and none in which the patient survived more over 7 years. A 72-year-old postmenopausal Japanese woman had been diagnosed with type C chronic hepatitis. Screening ultrasonography revealed a low echoic small nodular lesion located on S6 of the liver. Tiny hepatocellular carcinoma was diagnosed by needle biopsy and the patient was treated with percutaneous ethanol injection therapy. Two years later, she underwent a work-up because of an elevation in serum alpha-fetoprotein level, and whole body Computed Tomography scan revealed a large solid pelvic mass. At laparotomy, a large right ovarian tumor was revealed. No other lesion was found. A bilateral salpingo-oophorectomy was performed. On microscopic examination, the tumor showed predominantly solid and trabecular patterns. Pseudo-glandular patterns were also observed. The final pathological diagnosis was hepatocellular carcinoma metastatic to the right ovary. Postoperatively, the serum alphafetoprotein concentration decreased to within the normal range. Although, we resected an implantation in her right thoracic wall seven months post laparotomy, she did not show any evidence of recurrence during her 7 year follow up period. Metastatic hepatocellular carcinoma to the ovary is very rare. If a patient presents with findings similar to those describing our patient, the examining physician should be aware of the risk of potential ovarian metastases from hepatocellular carcinoma. Surgical resection may be the only effective treatment strategy for static ovarian cancer.
\end{abstract}

Keywords: Hepatocellular carcinoma; Metastatic neoplasm; Ovary

Abbreviations: AFP: Alpha Fetoprotein; HCC: Hepatocellular Carcinoma; HP-1: Hepatocyte Paraffin 1

\section{Introduction}

Hepatocellular Carcinoma (HCC) usually metastasizes to the lung, bone, adrenal glands, diaphragm and lymph nodes. In this study, we present a case of metastatic HCC to the ovary and review literature. To the best of our knowledge, only ten such cases have previously been reported: eight in English, one in Korean and one in Spanish [1-8]. The treatment has usually been surgery and chemotherapy. The survival time for almost all cases was about one year. Therefore, the prognosis of HCC patients with distant metastases tends to be extremely poor. We present a patient with metastatic HCC to the ovary who, at the time of this report, has had a disease-free survival of 7 years since last surgical resection.

\section{Case Report}

A 72-year-old postmenopausal Japanese woman had been diagnosed with type C chronic hepatitis. In 2001, screening ultrasonography revealed a low echoic $16 \times 17 \mathrm{~mm}$ nodular lesion located on S6 of the liver. Serum Alpha-Fetoprotein (AFP) concentration was $141 \mathrm{ng} / \mathrm{mL}$ (normal range 0-35 ng/mL). HCC was diagnosed by needle biopsy. She was treated with Percutaneous Ethanol Injection Therapy (PEIT) based on her wish for minimally invasive therapy. She underwent four PEIT procedures, after which there was no evidence of tumor and her serum AFP concentration decreased to within the normal range. Two years later, she underwent a close work-up because her serum AFP concentration rose to $98.906 \mathrm{ng} / \mathrm{mL}$ without any abnormalities in the liver. Gallium scintigraphy showed an abnormal accumulation in the pelvis, and Computed Tomography (CT) scan revealed a solid pelvic mass $12 \mathrm{~cm}$ in diameter (Figure 1). She was asymptomatic and her serum CA125 concentration was $12.2 \mathrm{U} / \mathrm{ml}$. Laparotomy revealed a right ovarian tumor with a small amount of ascites. The left ovary was intact, the liver surface was smooth, and no other lesion was found.
The histological diagnosis on a frozen section from the right ovarian tumor suggested metastatic HCC. Accordingly, a bilateral salpingooophorectomy was performed. On gross examination, the right ovarian tumor was approximately $12 \mathrm{~cm}$ and its cut surface revealed a yellowish solid tumor with necrosis and hemorrhage (Figure 2). On microscopic examination, the tumor showed a predominantly solid, trabecular pattern with eosinophilic cytoplasm (Figure 3). Pseudo-glandular patterns, nuclear atypia and mitoses were observed (Figure 4). Immunohistochemical staining showed that tumor cells were strongly reactive with AFP and hepatocyte paraffin 1 (HP1) antibody (Figures $5 a$ and $5 b$ ). The final pathological diagnosis was metastatic HCC to the

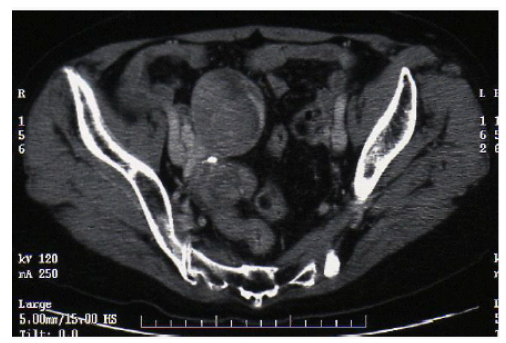

Figure 1: Abdominal CT scan showing a solid $11 \mathrm{~cm}$ diameter right adnexal mass.

*Corresponding author: Akimasa Fukui, Department of Obstetrics and Gynecology, Kurume University School of Medicine, 67, Asahimachi, Kurume Fukuoka, 830-0011, Japan, Tel: +81-942-31-7573; Fax: +81-942-35-0238; E-mail fakimasa@yahoo.co.jp

Received November 17, 2012; Accepted January 23, 2013; Published January 25, 2013

Citation: Fukui A, Ushijima K, Nishio S, Fujiyoshi K, Kage M (2013) Long Survival in a Rare Case of Hepatocellular Carcinoma that Metastasized to the Ovary: A Case Report. J Clin Case Rep 3: 249. doi:10.4172/2165-7920.1000249

Copyright: (c) 2013 Fukui A, et al. This is an open-access article distributed under the terms of the Creative Commons Attribution License, which permits unrestricted use, distribution, and reproduction in any medium, provided the original author and source are credited. 




Figure 2: The cut surface of the ovarian mass demonstrating a yellowish solid tumor with necrosis and haemorrhage.

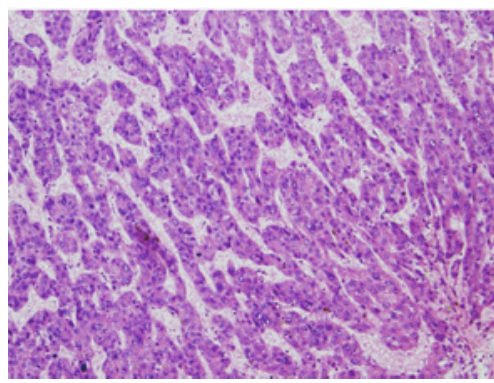

Figure 3: The tumor showed mainly solid, trabecular patterns with eosinophilic cytoplasm. Hematoxylin-eosin staining 100X.

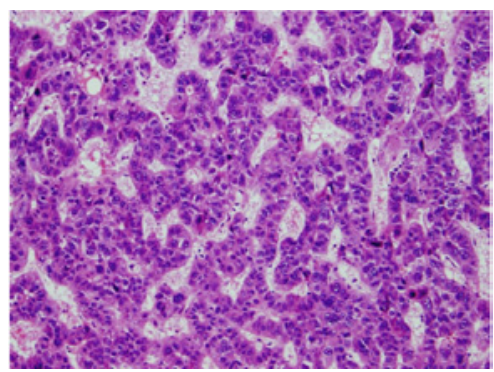

Figure 4: Pseudo-glandular patterns, mitoses and nuclear atypia were observed (100X).

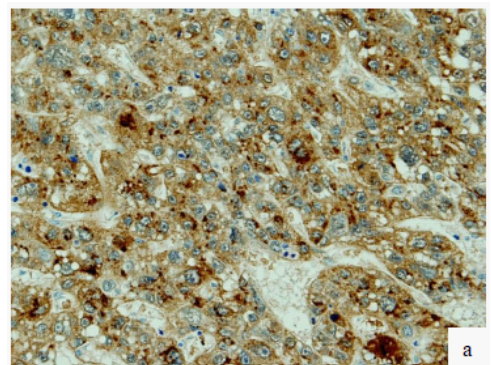

Figure 5a: Immunohistochemical staining showing strong reactivity of tumor cells with AFP (200X)

right ovary. She received no postoperative therapy. Postoperatively, the serum AFP concentration decreased to within the normal range. Seven months after surgery, CT showed a $7 \mathrm{~cm}$ right thoracic wall mass this tumor was an implantation of HCC, and was successfully resected. She has had no evidence of disease since operation 7 years ago.



Figure 5b: Immunohistochemical staining showing a strong reactivity of tumor cells with HP1 antibody (200X).

\section{Discussion}

Metastatic ovarian cancer most often originates from gastric or rectal cancer. The liver is an uncommon primary origin for metastatic ovarian cancer. Generally, HCC tends to invade the hepatic or portal veins, thereafter frequently metastasizing to the lung. Other common metastatic sites include bone, adrenal glands, diaphragm and lymph nodes [1]. However, because of improvements in survival from primary hepatic tumor, an increase in the number of metastases to the ovary has been observed [9].

In 1983, Oortman and Elliott was the first to report metastatic HCC to the ovary [2]. Table 1 summarizes the English-language case reports that we were able to find [1-6]. There was a wide range in the ages of patients (31-68 years old, mean 52). Our patient is the oldest. The ovary was the first metastatic site in all cases, and all but one had increased AFP concentration at the time their ovarian tumors were diagnosed. In four cases, the diagnoses of HCC and ovarian tumor were made simultaneously. Six of eight had unilateral tumors (three on the left side and three on the right side). The reported tumors ranged from solid and firm to soft and yellowish or greenish with focal necrosis and hemorrhage.

The differential diagnosis of metastatic HCC to the ovary includes hepatoid yolk sac tumor and primary hepatoid carcinoma [3]. Hepatoid yolk sac tumors tend to occur in young women and are associated with foci of a typical yolk sac or other germ cell elements. Such foci were not present in our case.

Ishikura and Scully first described primary hepatoid carcinoma of the ovary in 1987 [10]. It occurs in older, mostly postmenopausal, woman [3]. Although it belongs to the category of miscellaneous primary tumor, most cases are associated with surface epithelial type adenocarcinomas, suggesting the possibility of a surface epithelial origin [11]. Elevation of CA125 concentration, a marker for ovarian surface epithelial tumor, would support the diagnosis of primary ovarian cancer [12]. There was no association with a surface epithelial tumor in the present case and CA125 concentration was not elevated. Hepatoid carcinoma resembles HCC in both its histological features and immunoreactivity to AFP. HP1 is a monoclonal antibody that is highly specific for hepatocytes, but it is not useful for distinguishing primary ovarian hepatoid carcinoma from metastatic HCC. This is because its reactivity correlates with the degree of hepatic differentiation [13]. Tumor bilaterality, bile production or canaliculi also do not distinguish metastatic HCC from hepatoid carcinoma. CK19 and CK20 are expressed in normal gastrointestinal epithelial cells, bile duct cells, and some adenocarcinomas including ovarian surface epithelial carcinoma, but are not expressed in normal hepatocytes or hepatocellular carcinoma [12]. Unfortunately, we were unable 


\begin{tabular}{|c|c|c|c|c|c|c|}
\hline Study & Age & Chief complaint & AFP (ng/ml) & Tumor size $(\mathrm{cm})$ & Treatment & $\begin{array}{l}\text { Prognosis } \\
\text { (months) }\end{array}$ \\
\hline Oortman and Elliott [2] & 36 & AFP elevation & 18800 & $4 \times 6$ & $\mathrm{TAH}+\mathrm{BSO}$ & NR \\
\hline Young et al. [3] & 38 & AFP elevation & 14818 & INR & $\mathrm{TAH}+\mathrm{BSO}$ & DOD (55) \\
\hline Young et al. [3] & 31 & Back pain & 2700 & NR & $\mathrm{TAH}+\mathrm{BSO}$ & DOD (18) \\
\hline Young et al. [3] & 68 & Abdominal distention & 11000 & NR & BSO+omentectomy & NED (5) \\
\hline Khunamornpong et al. [4] & 56 & NR & & 15 & $\begin{array}{l}\text { TAH+BSO+sigmoid } \\
\text { colostomy }\end{array}$ & DOD (4) \\
\hline De Groot et al. [1] & 47 & None & 4 & 20 & BSO & DOD (12) \\
\hline Lee et al. [5] & 43 & AFP elevation & 336520 & 7 & RSO & NR \\
\hline Benito et al. [6] & 26 & NR & W.N.L & 14 & RSO+omentectomy & NED (12) \\
\hline Present case & 72 & AFP elevation & 98906 & 12 & BSO & $\operatorname{NED}(86)$ \\
\hline
\end{tabular}

This table showing 9 case reports of metastatic hepatocellular carcinoma to the ovary.

AFP: Alpha-fetoprotein; TAH: Total Abdominal Hysterectomy; BSO: Bilateral Salpingo-Oophorectomy; RSO: Right Salpingo-Oophorectomy; NR: Not Reported

Table 1: Metastatic hepatocellular carcinoma to the ovary.

to perform CK19 and CK20 immunohistochemical staining in this case. Kwon et al. said, however, it is extremely difficult to differentiate primary ovarian hepatoid carcinomas from metastatic hepatocellular carcinoma using any kind of ancillary study, with the exception of clinical identification of a hepatic tumor [14]. Considering the features of the tumor, we considered primary hepatoid carcinoma to be unlikely in this case.

The three modes of HCC spread are hematogenous metastasis, lymphatic metastasis and direct invasion. Of these, the most frequent is hematogenous metastasis [15]. In our case, there were no metastases to the liver, peritoneum, right ovary or other abdominal organs. There was no evidence of lymphovascular invasion of the HCC, and peritoneal washing cytology was negative at the time of operation for the ovarian tumor. These findings suggest that metastasis to the ovary was by hematogenous spread rather than lymphatic spread or seeding due to PEIT. However, the location of the right thoracic wall metastasis suggests that it resulted from peritoneal seeding due to PEIT.

The optimal treatment for ovarian metastases of HCC has not yet been established. Generally, the prognosis of HCC patients with distant metastases is extremely poor; most patients survive less than a year [16]. As there have only been ten cases reported in the literature, there is little information available regarding prognostic factors associated with outcomes in HCC metastatic to the ovary. Tumor grade, tumor size and patient age have not been reported to be associated with prognosis. In cases of HCC, well controlled primary lesion and complete surgical resection of the metastatic lesion has occasionally resulted in long term survival, as in our case. Wide-spread metastasis and incomplete resection may be associated with poor survival. Previous reports have indicated that chemotherapy is ineffective. Determining serum AFP concentration is useful for early detection of recurrence of HCC. In the present case, the patient's serum AFP concentration correlated closely with her disease status. We found her ovarian metastasis because of an increase in AFP concentration, which decreased after ovary removal.

\section{Conclusion}

Metastatic HCC to the ovary is very rare. The diagnosis is usually made based on clinical findings and AFP concentrations. If a patient presents with findings similar to those of our patient, the examining physician should be aware of the possibility of ovarian metastases from HCC. Surgical resection of the tumor may be the only effective treatment strategy.

\section{Acknowledgements}

The authors thank Yukio Tateishi who performed the medical treatment and provided clinical information. We are supported in part by the Supporting Fund of Obstetrics and Gynecology, Kurume University.

\section{References}

1. De Groot ME, Dukel L, Chadha-Ajwani S, Metselaar HJ, Tilanus HW, et al (2000) Massive solitary metastasis of hepatocellular carcinoma in the ovary two years after liver transplantation. Eur J Obstet Gynecol Reprod Biol 90: 109-111.

2. Oortman EH, Elliott JP (1983) Hepatocellular carcinoma metastatic to the ovary: a case report. Am J Obstet Gynecol 146: 715-717.

3. Young RH, Gersell DJ, Clement PB, Scully RE (1992) Hepatocellular carcinoma metastatic to the ovary: a report of three cases discovered during life with discussion of the differential diagnosis of hepatoid tumors of the ovary. Hum Pathol 23: 574-580.

4. Khunamornpong S, Siriaunkgul S, Chunduan A (1999) Metastatic hepatocellular carcinoma of the ovary. Int J Gynaecol Obstet 64: 189-191.

5. Lee JM, Park KM, Lee SY, Choi J, Hwang DW, et al. (2011) Metastasis of hepatocellular carcinoma to the ovary: a case report and review of the literature. Gut Liver 5: 543-547

6. Benito V, Segura J, Martínez MS, Arencibia O, Lubrano A (2012) Fibrolamellar hepatocellular carcinoma metastatic to the ovary. J Obstet Gynaecol 32: 200 202.

7. Kim TH, Cheung DY, Chung WB, Son DK, Jo DH, et al. (2004) A case of metastatic hepatocellular carcinoma of the ovary. Korean J Gastroenterol 43: 215-218.

8. Bilbao I, Vilallonga R, Allende E, Montero A, Quiroga S, et al. (2008) Krukenberg's tumor as the first clinical manifestation of fibrolamellar hepatocarcinoma Gastroenterol Hepatol 31: 341-346.

9. Itoh Y, Ohkubo K, luchi H, Michitaka K, Horiike N, et al. (2002) Chronological changes of causes of death and distant metastasis in hepatocellular carcinoma. Oncol Rep 9: 331-335.

10. Ishikura H, Scully RE (1987) Hepatoid carcinoma of the ovary. A newly described tumor. Cancer 60: 2775-2784.

11. Tochigi N, Kishimoto T, Supriatna Y, Nagai Y, Nikaido T, et al. (2003) Hepatoid carcinoma of the ovary: a report of three cases admixed with a common surface epithelial carcinoma. Int J Gynecol Pathol 22: 266-271.

12. Pandey M, Truica C (2011) Hpatoid carcinoma of the ovary. J Clin Oncol 29: 446-448.

13. Pitman MB, Triratanachat S, Young RH, Oliva E (2004) Hepatocyte paraffin 1 antibody does not distinguish primary ovarian tumors with hepatoid differentiation from metastatic hepatocellular carcinoma. Int J Gynecol Pathol 23: $58-64$

14. Kwon JE, Kim SH, Cho NH (2006) No ancillary finding is valid to distinguish a primary ovarian hepatoid carcinoma from metastatic hepatocellular carcinoma. Int J Gynecol Cancer 16: 1691-1694.

15. Kanda M, Tateishi R, Yoshida H, Sato T, Masuzaki R, et al. (2008) Extrahepatic metastasis of hepatocellular carcinoma: incidence and risk factors. Liver Int 28: $1256-1263$.

16. Stehlin JS Jr, de Ipolyi PD, Greeff PJ, McGaff CJ Jr, Davis BR, et al. (1988) Treatment of cancer of the liver. Twenty years' experience with infusion and resection in 414 patients. Ann Surg 208: 23-35. 